\title{
Crecimiento económico y medio ambiente
}

\author{
Adolfo Figueroa
}

RESUMEN

La relación entre crecimiento económico y medio ambiente es uno de los problemas de mayor interés en la economía moderna. Si bien hay cada vez más datos empíricos disponibles, las explicaciones teóricas siguen siendo tema de debate. En este trabajo se presenta un modelo teórico elemental de las interacciones entre el proceso económico y el medio ambiente, de acuerdo con la teoría de GeorgescuRoegen en la que las leyes de la termodinámica se incorporan al proceso económico. El modelo presupone que, actualmente, el proceso de crecimiento y distribución se efectúa bajo condiciones de estrés ambiental. Con este modelo se logra predecir y explicar las relaciones observadas entre crecimiento económico y medio ambiente. Asimismo, del modelo se desprenden nuevos temas de discusión en el ámbito de las políticas públicas. Por ello, con este artículo se procura contribuir al debate acerca de las alternativas sociales sobre el futuro de la humanidad.

PALABRAS CLAVE

CLASIFICACIÓN JEL

AUTOR
Crecimiento económico, medio ambiente, consumo, recursos no renovables, contaminación, termodinámica, aspectos económicos, aspectos ambientales

B52, E24, O13, O15, O33, Q32

Adolfo Figueroa es profesor emérito de Economía del Centro de Negocios (centrum) de la Pontificia Universidad Católica del Perú. afiguer@pucp.edu.pe 


\section{I}

\section{Introducción}

La relación entre el crecimiento y el medio ambiente ha sido estudiada tanto desde el punto de vista empírico como teórico. Los resultados más relevantes indican que la producción mundial por persona se incrementó a una tasa del 1,4\% anual entre los años 1870 y 2000, y registra el mayor crecimiento (4\%) en el último siglo (Maddison, 2003). A un ritmo similar, este proceso estuvo acompañado de la degradación del medio ambiente, sobre todo del agotamiento de los recursos naturales no renovables y la contaminación. De hecho, en algunos estudios se señala que ciertos recursos no renovables están a punto de agotarse (Clugston, 2012). La contaminación suele medirse en términos de concentración de dióxido de carbono $\left(\mathrm{CO}_{2}\right)$ en la atmósfera, medida en partes por millón (ppm). Según el físico Richard Muller, la cantidad de $\mathrm{CO}_{2}$ se mantuvo constante en $280 \mathrm{ppm}$ entre el año 800 de la era cristiana y los últimos años decimonónicos, pero en el último siglo trepó a 380 ppm, registrando un aumento del $36 \%$. Si se continúa quemando combustibles fósiles habrá aún más $\mathrm{CO}_{2}$. Entre las formas en que la actividad humana genera dióxido de carbono se destacan la quema de combustibles fósiles y la destrucción de extensas áreas forestadas (Muller, 2008, págs. 265 y 266).

También hay que tener en cuenta que la temperatura media del planeta no cesó de subir desde la revolución industrial que comenzó alrededor de 1850 (IPCC, 2007). Estimar si el recalentamiento del planeta y el cambio climático resultante son factores endógenos o exógenos del proceso económico todavía es tema de debate entre los científicos. Los factores que afectan al cambio se pueden sintetizar en tres premisas: primero, la quema de combustibles fósiles acrecienta la concentración de $\mathrm{CO}_{2}$ en el aire; segundo, el $\mathrm{CO}_{2}$ es un gas de efecto invernadero; tercero, el efecto invernadero incrementa la temperatura media del planeta. Las dos primeras premisas están aceptadas por la comunidad científica, pero la tercera está en discusión.

Según algunos científicos, las emisiones de gases de efecto invernadero son el origen del recalentamiento del planeta, lo que produce un cambio climático; es

$\square$ El autor desea expresar su gratitud a un árbitro anónimo de la Revista CEPAL, por sus valiosos comentarios, y también a Javier Vásquez de CENTRUM, por su excelente trabajo de asistencia en la preparación de este artículo. decir, la producción genera desechos y contaminación que provocan un cambio climático (Aeschbach-Hertig, 2007). De allí que dicho cambio se considere un factor endógeno del proceso de producción. Para otros científicos, el cambio climático es exógeno: se produce principalmente debido a variaciones naturales de la actividad solar (Chilingar, Sorokhtin y Khilyuk, 2008, pág. 1572). Un tercer grupo de científicos creen que, si bien el cambio climático es un problema complejo y difícil de descifrar con precisión, es endógeno, pero no en su mayor parte (IPCC, 2007, citado en Muller 2008, pág. 254).

En el caso particular de América Latina, existe evidencia empírica sobre la degradación del medio ambiente ocurrida en el largo período del crecimiento económico desde la década de 1940 (Sunkel y Gligo, 1980; Gligo 1993). También se ha mostrado que el efecto negativo del cambio climático en la producción media y la variabilidad de la producción constituye un conjunto de hechos estilizados (Galindo y Samaniego, 2010).

Estos son los hechos. Pero ¿cómo pueden explicarse? Desde el pensamiento económico estándar, el artículo de Robert Solow (1974) sigue siendo la referencia clásica; no obstante, como se verá más adelante, este planteamiento presenta problemas que la nueva literatura no ha logrado resolver (Barro y Sala-i-Martin, 2004; Grimaud y Rouge, 2005; Lafforgue, 2008). Nicholas GeorgescuRogen (1971) adoptó un enfoque diferente al introducir las leyes de la termodinámica en el proceso económico.

El objetivo del este estudio es presentar un modelo teórico en el marco de la teoría de Georgescu-Rogen. Mediante este modelo se procura dar cuenta de las interacciones entre el proceso económico y el medio ambiente, y establecer las interrelaciones entre el crecimiento de la economía y la dimensión medioambiental. Por otra parte, se intenta explicar los hechos descritos anteriormente y encontrar respuesta a los siguientes interrogantes: ¿Puede el crecimiento ser perpetuo? ¿Puede el consumo distribuirse equitativamente de generación en generación? ¿Cuál es el papel del progreso tecnológico en las interacciones entre crecimiento y degradación del medio ambiente?

El artículo se desarrolla de la siguiente manera: en la sección II se expone el modelo A, referido al proceso 
económico con recursos naturales no renovables. En la sección III se aborda la frontera intergeneracional del consumo. La sección IV se refiere a la introducción de las leyes de la termodinámica en el proceso económico, según el modelo B. La sección V concierne al modelo
$\mathrm{C}$, en que se contempla la sustitución entre capitaltrabajo y recursos naturales. En la sección VI se hace referencia a los factores que pueden generar cambios en la frontera intergeneracional del consumo. Por último, en la sección VII se ofrecen las conclusiones.

\section{II}

\section{Modelo A: el proceso económico con recursos naturales no renovables}

El modelo teórico presupone una sociedad humana abstracta con una dotación determinada de recursos y tecnología de producción. Esta sociedad produce un solo bien denominado "bien B".

En relación con el proceso de producción, en el modelo se utiliza el marco de flujos y fondos de GeorgescuRoegen (1971). Por definición, el proceso de producción es un proceso continuo que se reproduce a sí mismo y en el que se pueden distinguir dos categorías de factores de producción: los "fondos", que actúan como agentes, y los "flujos", que son los insumos que se transforman en bienes; en otras palabras, los fondos son factores del proceso de producción que ingresan y egresan, en tanto que los flujos solo pueden ingresar o egresar.

El algoritmo que se aplicará en la construcción del modelo más apropiado comienza con el modelo A, que presupone un proceso particular de producción en el que se produce el bien $\mathrm{B}$. Se lo representa bajo la forma de un sistema de producción de la siguiente manera:

$$
\mathrm{Q}_{\mathrm{j}}^{*}=\mathrm{F}(\mathrm{K}, \mathrm{L})
$$

$\mathrm{Q}_{\mathrm{j}}^{*}=(1 / \mathrm{z}) \mathrm{N}_{\mathrm{j}}$, tal que $\mathrm{z}>0$ y $\Sigma \mathrm{N}_{\mathrm{j}} \leq \mathrm{S}_{0}, \mathrm{j}=1,2, \ldots, \mathrm{T}$

El sistema productivo (1)-(2) presupone que el flujo de producción bruta $\mathrm{Q}^{*}$ se genera en el período $\mathrm{j}$ mediante el uso de cantidades de dos tipos de factores: el fondo de servicios de las existencias de capital $\mathrm{K}$ y mano de obra L —ecuación (1) — y el flujo de insumos $\mathrm{N}$ provenientes de las existencias de recursos naturales no renovables $S_{0}$, que aquí se denominarán recursos minerales y que serán los únicos insumos considerados -ecuación (2). El capital K está compuesto por el mismo bien B. Los recursos naturales renovables no serán tenidos en cuenta en esta instancia.
El sistema productivo (1)-(2) representa un proceso de producción particular, donde $\mathrm{Qj}^{*}$ es el flujo de producto del período j y $\mathrm{Nj}$ es el flujo de recursos minerales utilizados en dicho período. La producción puede repetirse período tras período si las existencias de $\mathrm{K}$ y L continúan fijas en el tiempo y si el flujo de minerales está garantizado hasta el agotamiento de las existencias de $\mathrm{S}_{0}$.

El sistema de producción (1)-(2) presupone una "tecnología de sustitución limitada"; es decir, el primero y el segundo tipos de factores no son intercambiables entre sí. Los recursos minerales no pueden ser reemplazados por capital o mano de obra; pero K y L sí son factores sustituibles, como se indica en la ecuación (1). Los recursos minerales ingresan en el proceso productivo en una proporción fija respecto de la producción bruta, representada con el coeficiente $\mathrm{z}$, que es tecnológicamente determinada.

Por último, el proceso de producción (1)-(2) también presupone que en las empresas que producen el bien B el período de trabajo tiene una cierta extensión y que la intensidad de trabajo está dada. El modelo supone condiciones de pleno empleo de la maquinaria y la mano de obra. En el análisis de largo plazo, que es el aplicable a este estudio, se utilizará una unidad de tiempo extensa, por ejemplo: la década.

Algunas de estas presunciones sufrirán modificaciones en la construcción de dos modelos alternativos que se presentarán más adelante. Las leyes de la termodinámica (sobre las relaciones entre la materia y la energía) se incluirán en el modelo $\mathrm{B}$, en tanto que en el modelo $\mathrm{C}$ se considerará la sustitución entre fondos y flujos. El modelo $\mathrm{B}$ resultará ser más adecuado que los modelos A y C.

En el modelo A se puede considerar por un momento que los recursos naturales son factores redundantes; por ello, la ecuación relevante que se aplica al proceso productivo será la ecuación (1). Por definición, la 
producción neta equivale a la producción bruta menos la depreciación del capital, que es la cantidad de bienes asignados a la reposición de $\mathrm{K}$. El término "reposición" hace referencia aquí a la cantidad del bien B necesaria para mantener constantes las existencias de K; esto implica garantizar la misma cantidad de existencias e igual cantidad de fondos de servicios período tras período.

Si el coeficiente de reposición de K es b, entonces $\mathrm{b}$ representa la cantidad de bienes $\mathrm{B}$ que se necesitan por unidad de K para mantener K constante. Luego, si se multiplica b por la cantidad de $\mathrm{K}$, arrojará la cantidad total de bienes $\mathrm{B}$ necesarios para reemplazar directamente el desgaste de la maquinaria y así mantener las existencias de $\mathrm{K}$ constantes período tras período.

La ecuación de reposición para un período j se puede expresar de la siguiente manera:

$$
\begin{aligned}
\mathrm{R}_{\mathrm{j}} & =\mathrm{b} \mathrm{K} \\
& =\mathrm{rQ}_{\mathrm{j}}^{*}, 0<r<1
\end{aligned}
$$

En la ecuación (3), R indica la reposición total, es decir, la cantidad total de bienes B necesarios para mantener constantes las existencias de K. De la ecuación (1) resulta que a K y L dados, se conoce la cantidad de producción bruta $\mathrm{Q}^{*}$. Por ello, el flujo de reposición $\mathrm{R}$ puede representarse como una proporción fija de $Q^{*}$, el coeficiente $r$. En un proceso que sea efectivamente productivo, el coeficiente $r$ debe ser inferior a uno.

El flujo de producción neta Q puede expresarse así:

$$
\begin{aligned}
& Q_{j}=Q_{j}^{*}-R_{j} \\
& =Q_{j}{ }^{*}-r Q_{j}{ }^{*} \\
& =(1-r) Q_{j}^{*}
\end{aligned}
$$

La ecuación (4) muestra que el flujo de Q es una proporción fija del flujo de Q*. Debido a que hay reposición, K es un factor "renovable" y la producción neta se sostiene a lo largo del tiempo; es decir, esta puede repetirse período tras período si los recursos minerales son redundantes. La producción neta puede asignarse a la acumulación de capital o al consumo; sin embargo, el modelo presupone que el total de dicha producción se asigna solo al consumo.

Si se considera que la sociedad cuenta con maquinarias y mano de obra en cantidades $\mathrm{K}_{1} \mathrm{y} \mathrm{L}_{1}$, que son ahora factores productivos redundantes, la ecuación relevante que se aplica al proceso de producción será la ecuación (2). Las existencias iniciales de recursos minerales $\mathrm{S}_{0}$ irán disminuyendo en forma continua durante el proceso productivo, aunque la cantidad de producción bruta sea igual período tras período. Por ello, la cantidad remanente de recursos minerales al final del período $\mathrm{T}$, indicado como $\mathrm{S}(\mathrm{T})$, puede expresarse de la siguiente forma:

$$
\begin{aligned}
\mathrm{S}(\mathrm{T}) & =\mathrm{S}_{0}-\Sigma \mathrm{N}_{\mathrm{j}}, \mathrm{j}=1,2, \ldots, \mathrm{T} \\
& =\mathrm{S}_{0}-\Sigma \mathrm{z} \mathrm{Q}_{\mathrm{j}}^{*}=\mathrm{S}_{0}-\mathrm{z} \Sigma \mathrm{Q}_{\mathrm{j}}^{*} \\
& =\mathrm{S}_{0}-\mathrm{z} \mathrm{Q}^{*} \mathrm{~T}
\end{aligned}
$$

La ecuación (5) señala que si la cantidad de producción bruta está determinada, las existencias iniciales de recursos minerales disminuirán con el tiempo a razón de $\mathrm{N}=\mathrm{z}$ Q* por unidad de tiempo. La cantidad resultante en el período $\mathrm{T}$ se convierte en $\mathrm{S}(\mathrm{T})$ según el número de períodos en que se repite el proceso de producción.

\section{III}

\section{La frontera intergeneracional del consumo}

En esta sección se tomarán en cuenta ambas ecuaciones del sistema productivo (1)-(2). $\mathrm{K}_{1}$ y $\mathrm{L}_{1}$ representarán la dotación de maquinarias y mano de obra que permite la producción bruta $\mathrm{Q}_{1}{ }^{*}$, y que torna redundantes las existencias iniciales de recursos minerales. De allí que los requisitos de recursos minerales para la producción neta Q, tal como se la define en la ecuación (4), se pueden incluir en la ecuación (5), obteniéndose la siguiente expresión:

$$
\begin{aligned}
\mathrm{S}(\mathrm{T}) & =\mathrm{S}_{0}-\mathrm{z} \mathrm{F}\left(\mathrm{K}_{1}, \mathrm{~L}_{1}\right) \mathrm{T} \\
& =\mathrm{S}_{0}-\mathrm{z} \mathrm{Q}_{1}^{*} \mathrm{~T} \\
& =\mathrm{S}_{0}-[\mathrm{z} /(1-\mathrm{r})] \mathrm{Q}_{1} \mathrm{~T} \\
& =\mathrm{S}_{0}-\mu \mathrm{Q}_{1} \mathrm{~T}, \text { donde } \mu=\mathrm{z} /(1-\mathrm{r})
\end{aligned}
$$

En la ecuación (6), la tasa de agotamiento de las existencias iniciales de recursos minerales se representa 
en términos de producción neta $\mathrm{Q}_{1}$. Las existencias de recursos minerales disminuyen con el tiempo a razón de $\mathrm{N}=\mu \mathrm{Q}_{1}$ por unidad de tiempo, donde $\mu$ representa el requisito tecnológico de recursos minerales por unidad de producción neta.

El período de agotamiento de los recursos minerales se puede calcular igualando la ecuación (6) al valor cero, es decir $\mathrm{S}_{0}=\mu \mathrm{Q}_{1} \mathrm{~T}$. Esta paridad muestra que una cantidad inicial de recursos minerales y un coeficiente tecnológico dados implican una producción total fija, sea para el presente o para el futuro. Si el producto neto se duplica, el número de períodos durante los cuales se puede producir se reducirá a la mitad. A mayor producción neta, menor cantidad de períodos en los que se puede repetir. Un nivel fijo de producto neto no puede sostenerse a perpetuidad.

Si se divide la ecuación (6) por $\mu$, se obtiene:

$$
\begin{gathered}
\mathrm{S}(\mathrm{T}) / \mu=\mathrm{S}_{0} / \mu-\mathrm{Q}_{1} \mathrm{~T} \\
\mathrm{Q}(\mathrm{T})=\mathrm{Q}_{0}-\mathrm{Q}_{1} \mathrm{~T}
\end{gathered}
$$

El término $\mathrm{Q}(\mathrm{T})$ muestra la trayectoria en el tiempo del producto neto, mientras que $\mathrm{Q}_{0}$ es la cantidad de producto neto del período inicial. La capacidad productiva de los recursos naturales disminuye con el tiempo a una tasa establecida por el valor de $\mathrm{Q}_{1}$. Esta capacidad productiva se ilustra en la ecuación lineal (7), en la que el flujo de producción neta $\mathrm{Q}_{1}$, determinado por las existencias de maquinarias y mano de obra, constituye la pendiente (negativa).

La ecuación (7) representa las restricciones que imponen los fondos y los flujos en la producción neta $\mathrm{Q}_{1}$, de manera que los recursos minerales constituyen inicialmente el factor redundante $\left(Q_{0}>Q_{1}\right)$. A medida que $\mathrm{Q}_{1}$ se repite período tras período, los recursos minerales disminuyen en forma constante e irrevocable, hasta que finalmente terminan agotándose. Si se considera $\mathrm{Q}(\mathrm{T})=0$, se puede determinar este período de agotamiento total, al que llamaremos T', en que la producción neta será equivalente a cero. El período T' expresaría, por lo tanto, la extinción de la sociedad humana. El período en que los recursos minerales dejarán de ser redundantes, al que llamaremos $\mathrm{T}^{*}$, también se puede determinar en forma sencilla si se considera $\mathrm{Q}(\mathrm{T})=\mathrm{Q}_{1}$. Resulta claro que $\mathrm{T}^{*}=\mathrm{T}^{\prime}-1$.

Se introducirá un nuevo supuesto en el modelo. La sociedad no dejará que la naturaleza determine el fin de su existencia y tomará medidas ante el posible riesgo de su extinción. Se presupone que la sociedad decidirá en el período $\mathrm{T}^{*}$ (es decir, cuando los recursos minerales ya no sean redundantes) extender la duración de las existencias remanentes de recursos minerales por más de un período, disminuyendo el nivel de consumo. En estas condiciones, las existencias de recursos minerales podrían estar disponibles por varios períodos adicionales de tiempo y serían utilizadas a una tasa estipulada según el nuevo nivel de consumo hasta su total agotamiento. Llamaremos $\mathrm{T}^{\mathbf{o}}$ a este período final estipulado por la sociedad, de manera que $\mathrm{T}^{*}<\mathrm{T}^{\prime}<\mathrm{T}^{\mathrm{o}}$.

En el gráfico 1 se representa la ecuación (7). El eje cartesiano horizontal indica el tiempo y el vertical, la producción neta. A niveles dados de $\mathrm{K}_{1} \mathrm{y} \mathrm{L}_{1}$, y un nivel tecnológico $\Pi_{1}$, se representa el flujo resultante de producción neta mediante el segmento $\mathrm{OA}$, es decir, $\mathrm{Q}_{1}=\mathrm{OA}$. La restricción que imponen los recursos naturales se ilustra en la línea MV. Las unidades de OM de producción neta podrían, en consecuencia, producirse inicialmente con el nivel dado de existencias de recursos minerales; por ello, los recursos minerales son, al comienzo, redundantes. Pero a medida que se repiten las unidades de OA de producción neta período tras período, las existencias de recursos minerales disminuyen hasta su total agotamiento, que ocurre en el período $\mathrm{T}_{\mathrm{B}}$ ' (período 6 en el gráfico 1). Esta es la naturaleza básica

GRÁFICO

\section{Frontera intergeneracional del consumo}

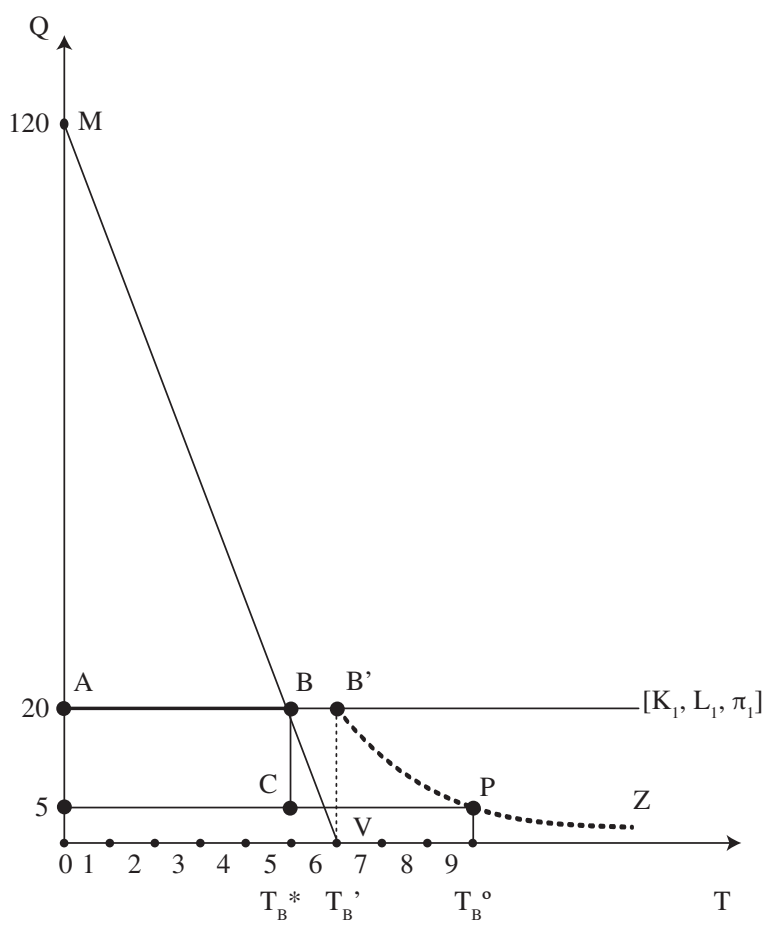

Fuente: elaboración propia.

Nota: En el eje cartesiano vertical se indican los niveles de consumo sobre la base de $\mathrm{OM}=120$ unidades y $\mathrm{OA}=20$ unidades; en el eje cartesiano horizontal se indican las generaciones a intervalos de tiempo. 
del proceso productivo de flujos y fondos, tal como se le representa en el sistema de producción (1)-(2).

También en el gráfico 1 se puede observar que la producción neta es equivalente al consumo. En el período $\mathrm{T}_{\mathrm{B}} *$ (período 5), cuando los recursos minerales dejan de ser redundantes, la sociedad decide intervenir y extender la duración de dichos recursos aminorando el consumo a una fracción del nivel de consumo actual OA. Los recursos minerales que no han sido utilizados al final del período podrían estar disponibles por varios períodos hasta su agotamiento irrevocable. Esto dependerá de las decisiones que tome la sociedad respecto del nivel de consumo. Si el nivel se reduce a la mitad, el tiempo se extenderá por dos períodos; si el nivel se acorta en un tercio, se extenderá por tres períodos, y así sucesivamente. Las posibilidades de niveles de consumo se ilustran en la hipérbola equilátera B'Z.

La trayectoria en el tiempo de las posibilidades de consumo puede denominarse "frontera intergeneracional del consumo". Esta frontera está representada por el segmento $\mathrm{AB}$ y por un punto particular del segmento B'Z. El segmento AB está limitado por las existencias de $\mathrm{K}_{1} \mathrm{y} \mathrm{L}_{1}$; y el segmento B'Z, por las existencias remanentes de recursos minerales.

Supóngase que la alternativa social elegida en el segmento B'Z sea el punto $P$. Más allá del período $T_{B}{ }^{*}$ (período 5), el nivel de consumo está establecido por el segmento CP (el nivel C equivale a un cuarto de OA en el gráfico 1) y se extenderá por cuatro períodos adicionales hasta el agotamiento de los recursos minerales en el período $\mathrm{T}_{\mathrm{B}}{ }^{\mathrm{o}}$ (período 9). La dotación inicial de maquinarias y mano de obra se torna aquí redundante y la cantidad de producción neta está limitada por la disponibilidad de los recursos minerales. En suma, se presupone que el número de trabajadores se mantiene igual, aunque solo una proporción del total de estos sea necesaria para la producción. Será indispensable introducir ciertos cambios institucionales en la sociedad para lograr una separación entre la participación de los trabajadores en la producción y en la distribución. Al no ser necesaria la reposición, las existencias de maquinarias podrán disminuir.

La distribución del consumo entre las distintas generaciones también se ilustra en el gráfico 1 . El nivel de consumo de la generación actual (OA para el período 1) será superior al consumo medio de las generaciones venideras (OA para cuatro generaciones y OC para cuatro generaciones). Como resultado, habrá desigualdad en el consumo entre generaciones. La razón proviene de la naturaleza finita de los recursos minerales, que impide que el nivel de consumo OA se mantenga en forma indefinida.

En conclusión, cuando en el proceso de producción se utilizan recursos naturales no renovables, el nivel de consumo de la generación actual no puede sostenerse a perpetuidad. Ello resulta del agotamiento irrevocable de tales recursos. Se destaca, asimismo, el grado de inequidad en el nivel de consumo entre generaciones: el nivel de consumo medio de las generaciones futuras será necesariamente inferior al de la generación actual.

\section{Modelo B: introducción de las leyes de la termodinámica en el proceso económico}

En la nueva disciplina de la economía ambiental se suelen reconocer dos escuelas de pensamiento (Hanley, Shogren y White, 2001). El pensamiento económico estándar en temas medioambientales se construye sobre la teoría neoclásica y la primera ley de la termodinámica. La otra escuela, conocida como "bioeconomía", fue fundada por Georgescu-Roegen (1971) e introdujo la segunda ley de la termodinámica — la ley de la entropía - en el proceso económico. Ambas leyes de la termodinámica se incluirán ahora en el análisis del proceso de producción, así como en el sistema de producción (1)-(2) y las relaciones de allí derivadas.
Hasta aquí, el efecto del consumo en el medio ambiente se vio reflejado en una constante disminución de las existencias de recursos minerales hasta su total agotamiento. A este proceso, se le denominará "efecto de agotamiento puro" de un recurso natural no renovable.

Georgescu-Roegen (1971, págs. 5 y 6) da una simple explicación de las leyes de la termodinámica que interesan al análisis del proceso económico. Se la puede resumir así:

"Tomemos el caso de una anticuada locomotora en la que el calor de la combustión del carbón fluye a la caldera y de esta a la atmósfera. Un resultado 
evidente de este proceso es un trabajo mecánico: el tren se ha desplazado de una estación a otra. Ahora bien, el proceso lleva también consigo otros cambios innegables; por de pronto, el carbón se ha transformado en cenizas. Con todo, algo es cierto: no se ha alterado la cantidad total de materia y energía. Esto es lo que dispone la Ley de la Conservación de la Materia y la Energía, que es la Primera Ley de la Termodinámica. Al principio, la energía química del carbón es libre, en el sentido de que está disponible para producir cierto trabajo mecánico. Sin embargo, en el proceso la energía libre pierde poco a poco su calidad. Finalmente, siempre se degrada por completo en el conjunto del sistema cuando se convierte en energía disipada, es decir, energía que ya no podemos emplear para el mismo propósito. En otras palabras, una entropía alta implica una estructura en la que la mayor parte de toda su energía es disipada, y una entropía baja una estructura en la que es cierto lo contrario.Esta es la Ley de la Entropía, que es la Segunda Ley de la Termodinámica; todo lo que dice es que la entropía del Universo (o de una estructura aislada) aumenta constantemente y de forma irrevocable. Podríamos decir que en el Universo hay una degradación cualitativa continua e irrevocable de energía libre en energía disipada".

En el resultado del proceso de producción se incluyen no solo bienes, sino también "males", ya que los desechos son también resultados irrevocables del proceso de producción. Esta limitación está contemplada en la primera ley de la termodinámica: la materia y la energía solo se transforman, no se pueden destruir ni generar.

La primera ley tiene otra relación con el proceso productivo. La producción de bienes materiales importa la transformación de insumos (el flujo es uno de los elementos de la producción) a través de los agentes (los fondos son el otro elemento). Por ello, los recursos minerales son "factores esenciales" en el proceso económico, en el sentido de que $\mathrm{N}=0$ significa $\mathrm{Q}^{*}=\mathrm{Q}_{1}=0$. Esta propiedad ya fue presentada como un supuesto del sistema productivo (1)-(2). Según la segunda ley, el desecho se transforma en contaminación del ambiente biofísico. El agotamiento de los recursos y la contaminación son dos formas de contribución del proceso económico a la degradación del medio ambiente.

La producción de bienes depende del medio ambiente por una doble vía: i) por ser una fuente de recursos minerales (entropía baja); y ii) por ser un reservorio para los desechos (entropía alta), que en conjunto degradan el medio ambiente (Daly, 1996, pág. 33). El tamaño finito de la Tierra impone límites a ambos elementos, ya que existe una cantidad dada de recursos minerales disponibles y una capacidad limitada para absorber los desechos, lo que significa que la capacidad de absorción del ecosistema es limitada para seguir albergando la vida humana, tal como la conocemos. Con respecto al proceso de producción, las existencias de recursos minerales no serían un problema si pudieran reciclarse, pero la ley de la entropía advierte sobre la imposibilidad del reciclaje absoluto; por otra parte, los desechos no serían un problema si la capacidad de absorción del ecosistema fuera infinita.

A consecuencia de ello, todo proceso productivo, incluso con una producción neta constante, implica un agotamiento continuo e irrevocable de los recursos minerales. De allí que el proceso económico sea una actividad humana que también puede verse como la transformación de la entropía baja (recursos minerales) en entropía alta (desechos y contaminación). La materia y la energía disponibles pueden utilizarse una sola vez en el proceso de producción; es decir, dicho proceso implica la degradación de energía libre en energía disipada.

Existe una estrecha interrelación entre ambas leyes de la termodinámica. El economista Kenneth Boulding (1976, pág. 5) señaló que, en un sistema cerrado, lo único que puede ocurrir con la primera ley es una reorganización; mientras que con la segunda ley la reorganización tiene lugar si existe el potencial que la haga posible y, a medida que la reorganización continúa, el potencial se va agotando hasta volverse nula, llegando a un punto en que nada más puede ocurrir.

En el proceso económico solo se reorganizan la materia y la energía, pero en él la capacidad productiva se degrada en términos cualitativos. Por ello, a medida que la producción se repite período tras período, el potencial del sistema productivo se degrada en forma continua e irrevocable. El proceso económico no es mecánico, sino entrópico.

¿Cómo afectan las leyes de la termodinámica a la frontera intergeneracional del consumo? En primer lugar, debe tenerse en cuenta el efecto de los desechos en la degradación cualitativa del ambiente biofísico. Los desechos provocan contaminación ambiental del agua, el aire y el suelo. Se debe presuponer que la contaminación eleva la temperatura media del planeta y que el clima afectará al proceso de producción tornándolo más riesgoso.

En segundo lugar, la contaminación es un resultado del proceso de producción; no obstante, tendrá un efecto de retroacción en dicho proceso, ya que aumentará el costo de reemplazo de las maquinarias. Debido al daño directo que la contaminación provoca en los bienes de capital y el mayor riesgo de destrucción por efecto 
del cambio climático, deberá considerarse una tasa de amortización mayor para mantener las maquinarias en condiciones productivas y duraderas.

En consecuencia, para conservar el mismo nivel de producción neta se necesitarán más recursos minerales. El flujo de contaminación se acumula en el medio ambiente, por ello cuando se obtiene la misma producción neta período tras período, el efecto de retroacción se acrecienta con el tiempo y aumenta también el coeficiente tecnológico de los recursos minerales necesarios por unidad de producción neta; es decir, el valor del coeficiente $\mu$ se va incrementando en forma constante.

El supuesto inicial sobre el proceso de producción indicada para el sistema (1)-(2) ahora tendrá que variar. Dados los valores $\mathrm{K}=\mathrm{K}_{1}, \mathrm{~L}=\mathrm{L}_{1}$ y $\Pi=\Pi_{1}$ se determinará el flujo de producción bruta $\mathrm{Q}^{*}=\mathrm{Q}_{1} *$, siempre que se disponga de los recursos minerales en cantidad $\mathrm{N}$, que ahora deben incluir los insumos materiales directos y los insumos indirectos necesarios según el nivel de contaminación (P). Este nivel en el período $\mathrm{T}$ puede expresarse de la siguiente forma:

$$
\mathrm{P}(\mathrm{T})=\Sigma \mathrm{P}_{\mathrm{j}}=\Sigma \beta \mathrm{N}_{\mathrm{j}}=\beta \mathrm{z} \Sigma \mathrm{Q}_{\mathrm{j}}^{*}, \mathrm{j}=1,2, \ldots, \mathrm{T}
$$

El coeficiente $\beta$ corresponde a la tasa de contaminación provocada por la quema de minerales en el proceso de producción o la tasa emisión de gases de efecto invernadero debido al uso de energía proveniente de recursos minerales.

Así, es posible determinar el coeficiente total de recursos minerales necesarios por unidad de producción neta. En primer lugar, los costos de reposición $\mathrm{R}$ de las maquinarias se expresan de la siguiente manera:

$$
\begin{aligned}
\mathrm{R}(\mathrm{T})= & \mathrm{r}_{1}^{*}+\mathrm{r}^{\prime} \mathrm{P}(\mathrm{T})=\mathrm{r} \mathrm{Q}_{1}^{*}+\mathrm{r}^{\prime} \beta \mathrm{z} \Sigma \mathrm{Q}_{\mathrm{j}}^{*} \\
= & \mathrm{r}_{1}^{*}+\mathrm{r}^{\prime} \beta \mathrm{z} \mathrm{Q}_{1}^{*} \mathrm{~T}=\left(\mathrm{r}+\mathrm{r}^{\prime} \beta \mathrm{z} \mathrm{T}\right) \mathrm{Q}_{1}^{*} \\
=\lambda(\mathrm{T}) \mathrm{Q}_{1}^{*} & \\
\mathrm{Q}(\mathrm{T}) & =\mathrm{Q}_{1}^{*}-\mathrm{R}(\mathrm{T}) \\
& =\mathrm{Q}_{1}^{*}[1-\lambda(\mathrm{T})],
\end{aligned}
$$

donde

$$
[1-\lambda]>0, \mathrm{y} \lambda^{\prime}(\mathrm{T})>0
$$

En la ecuación (9), el primer término es el costo típico de reposición, equivalente a la proporción $\mathrm{r}$ de la producción bruta total, más los costos de reposición derivados del efecto de la contaminación en las maquinarias, que equivale a la proporción ( $r^{\prime}$ ) del nivel de contaminación. De allí que el coeficiente de reposición total por unidad de producción bruta se representa con $\lambda$, que aumenta con el tiempo, y como una función de tiempo $\mathrm{T}$ se representa con $\lambda(\mathrm{T})$. La ecuación (10) ilustra la nueva relación entre la producción neta y la producción bruta.

La cantidad de recursos minerales que se necesitan por unidad de producción neta se obtiene a partir de la ecuación (10), de la siguiente manera:

$$
\begin{aligned}
\mathrm{N}(\mathrm{T}) & =\mathrm{z}_{1}^{*} \\
& =(\mathrm{z} /[1-\lambda(\mathrm{T})]) \mathrm{Q}_{1} \\
& =\varepsilon(\mathrm{T}) \mathrm{Q}_{1},
\end{aligned}
$$

donde $\varepsilon(\mathrm{T})=\mathrm{z} /[1-\lambda(\mathrm{T})], \varepsilon^{\prime}(\mathrm{T})>0, \mathrm{y} \varepsilon(0)=\mathrm{z} /(1-\mathrm{r})=\mu$

El coeficiente $\varepsilon$ representa la cantidad de recursos minerales que se necesitan por unidad de producción neta, en cuyo valor se incluye el efecto de retroacción de la contaminación en el proceso productivo. Por otra parte, el valor de este coeficiente aumenta con el tiempo porque el efecto de la producción en la contaminación es acumulativo.

Para derivar la trayectoria en el tiempo de la frontera de posibilidades del consumo, la ecuación (6) debe reformularse de modo de que incluya las nuevas relaciones resultantes:

$$
\begin{gathered}
\mathrm{S}(\mathrm{T})=\mathrm{S}_{0}-\Sigma \mathrm{N}_{\mathrm{j}}=\mathrm{S}_{0}-\mathrm{z} \Sigma \mathrm{Q}_{\mathrm{j}}^{*}=\mathrm{S}_{0}-\mathrm{z} \mathrm{Q}_{1}^{*} \mathrm{~T} \\
=\mathrm{S}_{0}-(\mathrm{z} /[1-\lambda(\mathrm{T})]) \mathrm{Q}_{1} \mathrm{~T} \\
=\mathrm{S}_{0}-\varepsilon(\mathrm{T}) \mathrm{Q}_{1} \mathrm{~T} \\
\mathrm{Q}(\mathrm{T})=\left[\mathrm{S}_{0} / \varepsilon(\mathrm{T})\right]-\mathrm{Q}_{1} \mathrm{~T}
\end{gathered}
$$

En la ecuación (12) se presenta la trayectoria en el tiempo de las existencias de recursos minerales, que al tiempo $\mathrm{T}$ son equivalentes a las existencias iniciales $\mathrm{S}_{0}$, menos la cantidad utilizada hasta ese período. La ecuación (13) es el resultado de la división de la ecuación (12) por el coeficiente $\varepsilon$, que aumenta con el tiempo.

En la ecuación (13) se muestra el proceso entrópico de producción, que incluye tanto los flujos como los fondos; también comprende las interacciones entre el proceso económico y el ambiente biofísico. En la cantidad de la producción neta $\mathrm{Q}_{1}$ —determinada por los fondos, las maquinarias y la mano de obra- están consideradas las limitaciones particulares de los recursos minerales. A medida que $\mathrm{T}$ aumenta, se incrementa la cantidad de recursos minerales por unidad de producción neta (el coeficiente $\varepsilon$ ), lo que implica un desplazamiento continuo 
hacia abajo del intercepto de la curva de la frontera. La trayectoria temporal de la frontera de producción, que está determinada por las limitaciones de los recursos minerales, ahora se torna no lineal y pasa a ser una curva convexa. A un mismo nivel de producción neta en el tiempo, se acelera el agotamiento de los recursos minerales respecto del caso anterior, cuando se había ignorado la retroacción de la contaminación en el proceso de producción.

En el gráfico 2 se representa el proceso entrópico de producción. El efecto del agotamiento se muestra en el panel (a). La línea recta MV representa un coeficiente tecnológico constante de los recursos minerales necesarios por unidad de producción neta (igual que en la frontera del gráfico 1). Esta línea representa el período inicial. Debido a que el coeficiente $\varepsilon$ aumenta con el tiempo, la línea MV tendrá una tendencia continua a moverse hacia adentro y así dibujar una frontera de producción que tiene la forma de una curva convexa, la curva MW. Esta frontera cortará el segmento $\mathrm{AB}$ en el punto $\mathrm{E}$. El proceso entrópico de producción conlleva una tasa de agotamiento más rápida de los recursos minerales, tal como indica la curva MW.

El período de intervención social ocurre cuando la curva MW corta la línea horizontal $\mathrm{AB}$ en el punto $\mathrm{E}$ en el período $\mathrm{T}_{\mathrm{d}}{ }^{*}$. Más allá de este período, las posibilidades

GRÁFICO 2

\section{Agotamiento y contaminación en el proceso de producción}

(a)

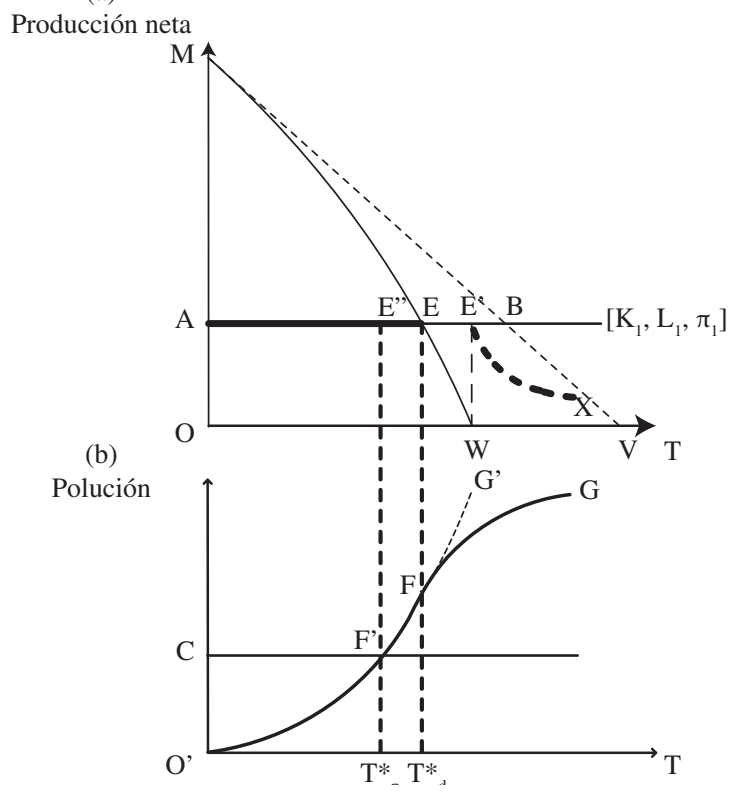

Fuente: elaboración propia. de consumo de los recursos minerales remanentes se expresarán en la curva E’X. Luego, la elección social determinará un punto particular a lo largo de esta curva. La frontera intergeneracional del consumo queda más limitada en comparación con la curva correspondiente del gráfico 1, debido al efecto de la ley de la entropía.

En el panel (b) del gráfico 2 se ilustra el efecto de la contaminación. A medida que se repite la misma cantidad de producción neta, el medio ambiente acumula más desechos y el nivel de contaminación es mayor, tal como representa la curva O'G. Por ello, la curva se eleva rápidamente hasta el período $\mathrm{T}_{\mathrm{d}}{ }^{*}$, momento en que los recursos minerales se tornan escasos. Más allá de este período, con la intervención social el nivel de contaminación sigue subiendo, pero a un ritmo más lento y en la trayectoria FG en lugar de la FG'; es decir, que los niveles de consumo descienden, la tasa de aumento de la contaminación también baja, pero el nivel de contaminación se incrementa en forma constante e irrevocable, como prevé la ley de la entropía. La sociedad puede modificar la tasa de degradación, pero no puede evitarla.

En el gráfico 2 es posible observar la acción de las dos leyes de la termodinámica. Estas leyes imponen limitaciones al proceso de producción por efecto del agotamiento y el efecto de la contaminación y, como se sugiere en el gráfico, estos efectos se interrelacionan. El efecto del agotamiento de los recursos minerales impone un límite temporal a una producción neta determinada: la producción OA puede repetirse hasta el período $\mathrm{T}_{\mathrm{d}}{ }^{*}$. La contaminación tendrá esta misma propiedad. Nuestro ecosistema tiene una capacidad limitada de absorción de desechos para seguir albergando la vida humana, la que se representa como un umbral, establecido por el nivel O'C, que ocurre en el período $\mathrm{T}_{\mathrm{p}}$ *. Si la contaminación atmosférica supera este umbral, la preservación de la vida humana, tal como la conocemos, no puede garantizarse. El segmento F'G de la curva ascendente de contaminación O'G indica una continua disminución en la calidad de vida de la sociedad debido a la contaminación de las fuentes de alimentos y el deterioro de la salud humana. Entonces será necesario adoptar ciertos cambios cualitativos y medidas de adaptación de los seres humanos; por ejemplo, los bajos niveles de oxígeno en el aire podrían generar un tipo de existencia humana anaeróbica.

Por efecto del agotamiento y la contaminación se impondrán diferentes umbrales temporales en la vida humana, según cuál de los dos efectos ocurra primero. En el gráfico 2, por ejemplo, el modelo presupone que el umbral de contaminación $\left(\mathrm{T}_{\mathrm{p}}{ }^{*}\right)$ se alcanzará antes del de agotamiento $\left(\mathrm{T}_{\mathrm{d}}^{*}\right)$. En este caso, la restricción 
del medio ambiente que debe considerarse es la capacidad del sistema ecológico de albergar a la sociedad humana, no el agotamiento de los recursos minerales. La capacidad ecológica sería el elemento esencial de escasez en el proceso económico. Todo puede producirse o reemplazarse, menos la capacidad ecológica. Los humanos no somos capaces de producir otro ambiente ecológico para sostener nuestra propia existencia. En este caso, la frontera intergeneracional del consumo solo transitará la trayectoria AE". El modelo presupone que la sociedad humana buscará tomar medidas frente al riesgo de su extinción. En dicha situación, los seres humanos deberán incorporar innovaciones tecnológicas e institucionales para ingresar en otra edad. Así como los humanos abandonaron la edad de piedra, aunque las piedras siguieron existiendo, los recursos minerales dejarán de utilizarse antes de su agotamiento total.

En el gráfico 2 se deja en claro que los retos ecológicos que enfrenta la humanidad seguirán existiendo aunque los niveles de consumo se mantengan constantes, es decir, aun si viviéramos en una sociedad sin crecimiento, ni de producción ni de población, e incluso en una sociedad con índices de crecimiento cero de producción y población. Por cierto, el problema se agravará si la sociedad decide emprender un proceso de crecimiento económico, como se verá más adelante.

El papel de los recursos naturales renovables en el proceso económico ha sido ignorado en el modelo entrópico. Implícitamente, el modelo ha supuesto que estos recursos eran abundantes, supuesto que ahora se revisará. Con tal fin, se distinguirán dos fuentes de energía del proceso de producción: i) la existencia de recursos minerales finitos y escasos de la corteza terrestre; ii) la energía solar que irradia directamente sobre la Tierra y provee una fuente de energía renovable para los recursos naturales, como los forestales e ictícolas.

La Tierra es un sistema termodinámico cerrado, en la medida en que obtiene la energía del Sol sin intercambio de materia con el espacio exterior (Baumgärtner, 2004, pág. 320). La escasez de los recursos naturales renovables proviene de las dimensiones limitadas de la Tierra, que actúa como una trampa de la energía solar. Por ser limitado y estar expuesto a la erosión, el suelo cultivable pertenece a la categoría de los recursos no renovables.

Los recursos ictícolas y forestales, entre otros recursos biológicos, pueden agotarse si la tasa de reposición biológica es inferior a la tasa de explotación humana. Cuando los recursos naturales renovables no se renuevan, comienzan a agotarse de igual forma que los recursos minerales. En este caso, los recursos naturales renovables pueden también incluirse en los coeficientes que determinan la frontera intergeneracional del consumo considerada en el modelo. Aquellos recursos naturales renovables que sí se renuevan pueden considerarse factores redundantes en el marco del modelo y pueden ignorarse.

En el panel (a) del gráfico 2, la frontera de la producción limitada por la energía solar, considerada como un factor redundante absoluto de la producción, puede representarse con una línea horizontal que parte de un punto ubicado por sobre el punto M. Bajo esta premisa, el segmento AE y la elección social de uno, y solo uno, de los puntos del segmento E'X pueden seguir representando la frontera intergeneracional del consumo, que pasa a estar determinada por los recursos no renovables y por aquellos recursos renovables que, debido al efecto de la actividad productiva humana, se convirtieron en no renovables.

\section{V}

\section{Modelo C: modelo que contempla la sustitución entre fondos y flujos}

En el pensamiento económico estándar se considera otro conjunto de supuestos sobre el proceso de producción, que se agrupan bajo el concepto conocido como "función de producción", y que se representa de la siguiente manera:

$$
\mathrm{Q}=\mathrm{F}(\mathrm{K}, \mathrm{L}, \mathrm{N})
$$

Esta corriente de pensamiento presupone que la cantidad de producción depende de la existencia de maquinarias, mano de obra y recursos naturales, y que todos estos factores son sustituibles entre sí (Solow, 1974, pág. 34). Esta ecuación simple implica además que los tres factores cumplen la misma función en el proceso de producción. De esta manera, sería factible afirmar que 
solo con maquinarias y trabajadores se puede obtener un nivel de producción neta, el que podría reproducirse eternamente. Nótese la diferencia con el enfoque de los flujos y los fondos, que estaba representado como un "sistema de producción" en las ecuaciones (1)-(2), en lugar de la función de producción.

Una consecuencia de la presunción del pensamiento económico estándar respecto del proceso productivo, es que el proceso para obtener una cantidad dada de producto neto puede repetirse por siempre. Así pues, el producto podría crecer eternamente, ya que no existen límites para la producción de bienes. Esta postura fue sostenida por Solow en su clásico artículo de 1974 y por Lafforgue (2008, pág. 541), quien expresa la visión moderna en forma resumida de la siguiente manera:

"Está ampliamente aceptado que las reservas finitas de recursos naturales no renovables no imponen necesariamente un límite al crecimiento. En particular, de la teoría neoclásica se desprenden tres posibilidades principales: i) sustitución del recurso con otros insumos, como el capital; ii) incremento de la eficiencia del recurso; y iii) desarrollo de tecnologías de apoyo. Sin cambios tecnológicos, sin embargo, ninguna de estas posibilidades permitirá compensar el agotamiento del recurso y continuar sosteniendo un crecimiento positivo en el largo plazo".

De acuerdo con esta corriente de pensamiento, una forma de introducir la sustitución entre las maquinarias y los recursos minerales sería suponer que el coeficiente tecnológico de los recursos minerales por unidad de producción neta puede disminuir a medida que aumenta el nivel de dotación de maquinarias en la sociedad. Esta sustitución sería posible gracias a las variaciones de los precios relativos de los minerales, es decir, cuando los recursos minerales se tornan más onerosos.

Incluso si se acepta la posibilidad de la sustitución, la cuestión queda sin resolver: ¿De dónde vendrían las nuevas maquinarias? Tendrían que producirse y, para ello, se necesitarían más recursos minerales. El efecto neto de la sustitución y el ahorro de los recursos minerales sería inferior que el efecto que la sustitución pura sugiere. Un ejemplo de ello son los molinos de viento que se utilizan en lugar de los generadores de energía a gasolina, ya que se requiere de minerales y otros insumos para su producción. Por otra parte, la producción neta es un bien material, que no puede desmaterializarse por completo según la primera ley de la termodinámica e impone un límite a las posibilidades de sustitución.

Según el gráfico 1, si una cantidad de capital puede sustituir recursos minerales, la línea MV tendría un tendencia a desplazarse hacia afuera, hacia otra línea (que podríamos dibujar con la nomenclatura M"V"). No obstante, producir esa cantidad de capital requeriría utilizar recursos minerales y también generaría costos de reposición en términos de dichos recursos. Por ello, el efecto neto de la sustitución de minerales sería inferior que el efecto inicial (un cambio de la línea MV a, por ejemplo, la línea M'V', que se dibujaría por debajo de la línea M"V"). Si se considera que el efecto neto es positivo, la curva de la frontera intergeneracional del consumo tendría una tendencia a desplazarse hacia afuera. De allí que el período $T^{*}$ se ampliaría, pero seguiría siendo finito. La sustitución podría ser mayor, pero siempre alcanzaría su límite. Si la línea MV representara el límite de las posibilidades de sustitución, el modelo contemplaría de este modo el efecto de la sustitución.

En suma, en el proceso entrópico de producción, la sustitución entre fondos y flujos es posible, pero solo en cierta medida. Esto obedece al supuesto de que los recursos minerales son factores esenciales de la producción, en concordancia con las leyes de la termodinámica. Sin embargo, los efectos de la sustitución no eliminarán la frontera intergeneracional del consumo. Incluso en condiciones posibles de sustitución, mientras un nivel dado de producción neta se repita período tras período, los recursos minerales se tornarán necesariamente escasos y se agotarán, en tanto que la contaminación aumentará. En consecuencia, en la medida en que los recursos minerales sean factores esenciales del proceso de producción, esta conclusión podrá sostenerse como verdadera; por lo tanto, las conclusiones que surgen del modelo entrópico B conservan su vigencia.

En comparación, en el pensamiento económico estándar se ha desarrollado una amplia literatura sobre la teoría del crecimiento. En los modelos presentados en reconocidos manuales de economía se predice que el crecimiento económico puede sostenerse eternamente y que la función de los recursos naturales no renovables puede ignorarse (Barro y Sala-i-Martin, 2004). Dadas la tasa de ahorro y las tasas de crecimiento demográfico y de la tecnología de la sociedad, y suponiendo que $\mathrm{K}$ crece al 5\%, L al 2\% y $\Pi$ al $3 \%$, el equilibrio dinámico implica que el producto total crecerá al 5\%; por lo tanto, el producto por persona crecerá al 3\% (la diferencia entre $5 \%$ y $2 \%$ ), y podrá hacerlo período tras período, indefinidamente. Así, en estos modelos no existen límites al crecimiento.

En algunos modelos de la teoría económica neoclásica se contemplan los recursos naturales no renovables y, en muy pocos, la cuestión de la contaminación, considerada apenas un problema de externalidades 
que puede resolverse sencillamente con los impuestos pigouvianos (Grimaud y Rouge, 2005). Los modelos neoclásicos en que se incluyen los recursos naturales en el proceso económico no dejan de ser mecánicos e ignoran las consecuencias cualitativas del crecimiento económico en el medio ambiente (por aplicación de la ley de la entropía). En este sentido, un investigador ha sostenido que la teoría neoclásica supone que las restricciones que imponen las leyes de la termodinámica al proceso económico son, en su conjunto, simplemente irrelevantes para el análisis económico (Baumgärtner 2004, pág. 308).

\section{VI}

\section{Cambios en la frontera intergeneracional del consumo}

La frontera intergeneracional del consumo se construyó sobre la base de un conjunto de condiciones dadas. Como variables exógenas del modelo B se incluyen la tecnología y la dotación de maquinarias, mano de obra y recursos minerales. Cabe analizar ahora el efecto que los cambios en estas variables exógenas ejercen en la frontera intergeneracional del consumo.

Un aumento exógeno en las existencias de maquinarias y mano de obra, junto con los cambios tecnológicos incorporados en las nuevas inversiones de bienes de capital y recursos humanos, causará un incremento del flujo corriente de la producción bruta y la producción neta; por ello, el nivel de consumo de la generación corriente también se acrecentará. En consecuencia, la tasa de agotamiento de las existencias de recursos minerales también tenderá a subir, impulsando el ascenso de la tasa de contaminación. En el gráfico 2, las mayores cantidades de K y L, así como un mayor nivel de П, modificarán la frontera intergeneracional del consumo de la siguiente manera: el nivel de consumo OA se desplazará hacia arriba, lo que conllevará un desplazamiento hacia adentro de la curva de agotamiento de recursos minerales MW y un desplazamiento hacia arriba de la curva de contaminación O'G; por lo tanto, los períodos críticos T’ y T* se acortarán.

Otra consecuencia es que el grado de desigualdad intergeneracional del consumo será mayor: el nivel de consumo de la generación actual aumentará, pero el nivel del consumo medio de las generaciones futuras decaerá. En otras palabras, el crecimiento económico implica un incremento de la desigualdad intergeneracional. Por consiguiente, la única elección que le queda a la sociedad es determinar la forma en que se distribuirá el nivel de consumo, y la correspondiente distribución de los recursos no renovables en una perspectiva intergeneracional. Un nivel de consumo mayor asignado a la generación actual implicará que esta utilizará más recursos minerales en detrimento de las generaciones futuras, las que dispondrán de tales recursos en menor cantidad y tendrán un nivel de consumo total más bajo.

Considérese a continuación un cambio tecnológico exógeno que genera la disminución del coeficiente inicial de recursos minerales por unidad de producción neta, el coeficiente $\varepsilon$, que está determinado por los coeficientes iniciales z y $\lambda$, tal como se ilustra en la ecuación (11). Toda reducción del valor de este coeficiente tecnológico es equivalente a un aumento de las existencias iniciales de recursos minerales. Este tipo de cambio tecnológico es ahorrador de recursos minerales. Por lo tanto, la curva de la frontera intergeneracional del consumo se desplazará hacia afuera y la curva de contaminación lo hará hacia abajo.

En el gráfico 2 se pueden visualizar estos efectos. Con las nuevas tecnologías que permiten ahorrar recursos minerales por unidad de producción neta, el intercepto de la curva de agotamiento de los recursos naturales se moverá desde M hasta un punto superior; por ello, la curva MEW se desplazará hacia afuera, al igual que la frontera intergeneracional del consumo. La curva de contaminación O'G se moverá hacia abajo. En las ecuaciones (8) y (10) se puede observar que el motivo de este desplazamiento es que la curva O'G está determinada por el flujo de la producción neta $\left(\mathrm{Q}_{1}=\mathrm{OA}\right)$, que permanece sin cambios, y también por los coeficientes tecnológicos, que disminuirán. Como resultado, los períodos críticos T' y T* se alargarán.

Sin embargo, no deja de ser cierto que el nivel actual de consumo no puede repetirse período tras período a perpetuidad. El progreso tecnológico, en consecuencia, no puede eliminar la frontera intergeneracional del consumo y solo puede moverla a un nivel diferente. En cada nuevo nivel de la tecnología habrá una nueva 
frontera intergeneracional del consumo. Asimismo, esta nueva frontera reducirá el grado de desigualdad entre las generaciones. Esta situación se produce si se considera que el cambio tecnológico no tiene costo. Si se tomara en cuenta que en la actividad de investigación y desarrollo (I+D) también se utilizarán recursos minerales, el efecto neto del progreso tecnológico sería menor.

¿Es posible que un cambio tecnológico sea suficientemente dinámico en el ahorro de recursos minerales como para alcanzar que un nivel de consumo se pueda repetir indefinidamente? En el supuesto de que el cambio tecnológico fuera considerado como una variable endógena que no tiene costo, se podría imaginar que si la mitad de los recursos minerales se agotara en un período dado de producción, el cambio tecnológico podría producirse inmediatamente y se reduciría a la mitad el coeficiente tecnológico de los minerales por unidad de producción neta, lo que equivale a duplicar los recursos minerales. Como consecuencia de ello, las existencias de recursos minerales permanecerían constantes en el tiempo, es decir, los dichos recursos se podrían convertir en recursos naturales "renovables". De allí que el nivel de consumo OA del gráfico 2 podría repetirse por siempre. A lo largo de esta línea horizontal, las maquinarias y los minerales se convertirían en recursos renovables gracias al cambio tecnológico.

Sin embargo, el panel inferior del gráfico 2 también debe ser considerado: el efecto de la contaminación seguirá siendo inevitable. Los recursos minerales serán utilizados en la producción $\mathrm{Q}_{1}$ en el primer período. $\mathrm{Y}$

\section{VII}

\section{Conclusiones}

De los tres modelos teóricos presentados en este artículo, en el B, que es un modelo entrópico, se incorporan las leyes de la termodinámica al proceso económico y se presta atención a las interacciones entre el proceso económico y el ambiente biofísico. Entre las interacciones se incluyen la trayectoria del consumo, el agotamiento de los recursos naturales y la contaminación ambiental. La trayectoria del consumo adopta la forma de una frontera intergeneracional: un nivel de consumo particular solo puede mantenerse durante un número finito de períodos.

Como variables exógenas del modelo entrópico se incluyen las existencias iniciales de capital, mano de obra y recursos minerales, junto con la tecnología. aunque se recuperen económicamente las existencias de dichos recursos mediante el cambio tecnológico, al ser utilizados generarán contaminación. En el siguiente período, la producción neta se repetirá y se utilizarán nuevos recursos minerales, y aunque se recuperen económicamente las existencias de tales recursos, el efecto de la contaminación no podrá evitarse, acumulándose por dos períodos, y así sucesivamente. La curva O'G se convertirá en una línea recta. En este caso, la contaminación, no el agotamiento, será el factor restrictivo del proceso económico. Mediante el cambio tecnológico se deberá ahora intentar contrarrestar el nivel de contaminación para alcanzar un proceso de producción no entrópico y resolver así dos problemas: el agotamiento de los recursos naturales y la contaminación. En las condiciones más favorables posibles, es improbable que el cambio tecnológico pueda revertir las leyes de la termodinámica.

El crecimiento económico combinado con el cambio tecnológico orientado al ahorro de los recursos minerales parecería presentar una ambigüedad respecto de los cambios en los umbrales temporales T' y $\mathrm{T}^{*}$. El efecto del crecimiento reduce la extensión de los períodos, pero el efecto de la tecnología los extiende. Sin embargo, en vista del argumento esgrimido anteriormente sobre los límites del cambio tecnológico, el efecto del crecimiento económico predominará y los umbrales temporales se acortarán. En suma, el crecimiento económico implica que la supervivencia de la sociedad humana, tal como la conocemos, tendrá una duración más corta.

Aumentos en las dotaciones del capital y en la mano de obra, así como nuevas tecnologías que ahorran mano de obra, reducen el número finito de períodos de la frontera intergeneracional del consumo, mientras que el progreso tecnológico que ahorra recursos naturales no renovables lo incrementa.

El modelo entrópico permite predecir las relaciones observadas entre el crecimiento económico y la degradación del medio ambiente. La observación empírica de la aceleración de las concentraciones de $\mathrm{CO}_{2}$ en el mundo que ha acompañado al período de rápido crecimiento económico, como se mostró en la Introducción, tiene así una explicación científica. 
El modelo entrópico tiene ciertas implicancias para las políticas públicas. En primer lugar, debido a que cualquier nivel de consumo puede mantenerse solo por ciertos períodos, un nivel mayor de consumo acortará el número de períodos; es decir, el crecimiento económico no puede ser perpetuo. En segundo lugar, el crecimiento de la economía acrecienta la desigualdad en la distribución intergeneracional del consumo. En tercer lugar, el progreso tecnológico solo puede reducir la tasa a la que se degrada el medio ambiente, pero la degradación es continua e irrevocable. En cuarto lugar, existe un conflicto de crecimiento no solo entre generaciones, sino también dentro de la generación actual: los países menos desarrollados tendrán un espacio ecológico más limitado para crecer si los países más desarrollados y los pocos países emergentes siguen creciendo. En este sentido, Edward Wilson, un biólogo de Harvard, concluyó que para que todo el mundo alcance el nivel de vida actual de los Estados Unidos de América con la tecnología existente se necesitarían dos planetas Tierra más (Wilson, 1998, pág. 282).

En el debate sobre las políticas públicas, tanto en el ámbito nacional como internacional, no se pueden ignorar estas relaciones. Estos son los problemas económicos fundamentales de nuestra era. La razón radica en que el proceso económico de crecimiento y distribución se realiza hoy día bajo condiciones de fragilidad ambiental, tal como lo explica el modelo entrópico presentado en este trabajo.
Aeschbach-Hertig, W. (2007), "Rebuttal of "On global forces of nature driving the Earth's climate. Are humans involved? by L.F. Khilyuk and G.V. Chilingar", Environmental Geology, vol. $52, \mathrm{~N}^{\circ} 5$, Springer.

Barro, R. y X. Sala-i-Martin (2004), Economic Growth, Cambridge, Massachusetts, The MIT Press.

Baumgärtner, S. (2004), "The Inada conditions for material resource inputs reconsidered", Environmental and Resource Economics, vol. 29, $\mathrm{N}^{\mathrm{o}} 3$, European Association of Environmental and Resource Economists.

Boulding, K. (1976), "The great laws of change", Evolution, Welfare, and Time in Economics, A. Tang, F. Westfield y J. Morley (eds.), Lexington, Massachusetts, Lexington Books.

Chilingar, G.V., O.G. Sorokhtin y L.F. Khilyuk (2008), "Response to W. Aeschbach-Hertig rebuttal of 'On global forces nature driving the Earth's climate. Are humans involved?", Environmental Geology, vol. 54, № 7, Springer.

Clugston, C. (2012), Scarcity. Humanity's Final Chapter, Bradenton, Florida, BookLocker.

Daly, H. (1996), Beyond Growth: The Economics of Sustainable Development, Boston, Massachusetts, Beacon Press.

Galindo, L.M. y J. Samaniego (2010), "La economía del cambio climático en América Latina y el Caribe: Algunos hechos estilizados", Revista de la CEPAL, N 100 (LC/G.2442-P), Santiago de Chile, abril.

Georgescu-Roegen, N. (1971), The Entropy Law and the Economic Process, Harvard University Press.
Gligo, N. (1993), "Environment and natural resources in Latin American development", Development from Within. Toward a Neostrucuturalist Approach for Latin America, O. Sunkel (ed.), Boulder, Colorado, Lynne Rienner Publishers.

Grimaud, A. y L. Rouge (2005), "Pollution, non-renewable resources, innovation, and growth: welfare and economic policy", Resource and Energy Economics, vol. 27, $\mathrm{N}^{\mathrm{o}} 2$, Amsterdam, Elsevier.

Hanley, N., J. Shogren y B. White (2001), Introduction to Environmental Economics, Oxford, Reino Unido, Oxford University Press.

IPCC (Grupo Intergubernamental de Expertos sobre el Cambio Climático) (2007), The Physical Science Basis, Ginebra, Secretaría del IPCC.

Lafforgue, G. (2008), "Stochastic technical change, non-renewable resource and optimal sustainable growth", Resource and Energy Economics, vol. 30, № 4, Amsterdam, Elsevier.

Maddison, A. (2003), The World Economy: Historical Statistics, París, Organización de Cooperación y Desarrollo Económicos (OCDE).

Muller, R. (2008), Physics for Future Presidents, Nueva York, Norton \& Company.

Solow, R. (1974), "Intergenerational equity and exhaustible resources", Review of Economic Studies, vol. 41, Oxford University Press.

Sunkel, O. y N. Gligo (eds.) (1980), Estilos de desarrollo y medio ambiente en América Latina, vol. 1, México, D.F., Fondo de Cultura Económica.

Wilson, E. (1998), Consilience. The Unity of Knowledge, Nueva York, Alfred Knopf. 